\title{
The role of immune checkpoint inhibitors (ICI) in the treatment of metastatic non-small cell lung carcinoma in the elderly
}

\author{
Johan Chan, Ravindran Kanesvaran \\ National Cancer Centre Singapore, 11, Hospital Drive, Singapore 169610, Singapore \\ Correspondence to: Ravindran Kanesvaran, MD. National Cancer Centre Singapore, 11, Hospital Drive, Singapore 169610, Singapore. \\ Email: ravindran.kanesvaran@singhealth.com.sg. \\ Provenance: This is an invited article commissioned by the Section Editor Dr. Jianrong Zhang (Incoming PhD Candidate, Centre for Cancer \\ Research, Faculty of Medicine, Dentistry and Health Sciences, University of Melbourne; Victorian Comprehensive Cancer Centre, Melbourne, \\ Victoria, Australia). \\ Comment on: Nosaki K, Saka H, Hosomi Y, et al. Safety and efficacy of pembrolizumab monotherapy in elderly patients with PD-L1-positive \\ advanced non-small-cell lung cancer: Pooled analysis from the KEYNOTE-010, KEYNOTE-024, and KEYNOTE-042 studies. Lung Cancer \\ 2019;135:188-95.
}

Submitted Oct 27, 2019. Accepted for publication Dec 12, 2019.

doi: $10.21037 / \mathrm{atm} .2019 .12 .76$

View this article at: http://dx.doi.org/10.21037/atm.2019.12.76

Immune checkpoint inhibitors (ICI) have had significant impact on the treatment of metastatic lung cancer patients with initial pivotal studies showing survival benefit in second line setting favoring ICI use compared to chemotherapy (14). Subsequently, ICI has shown a benefit in first line setting with single agent pembrolizumab (5) or in combination with chemotherapy based on PD-L1 expression results (6-9).

The incidence of lung cancer is strongly related to age and it is estimated that about $60.4 \%$ of newly diagnosed lung cancer patients are 65 years and older (10). Paradoxically, elderly patients are often underrepresented in trials despite the high prevalence of malignancies (11). A recent survey on health-related quality of life in elderly patients with cancer incorporated pooled data from 25 EORTC trials involving more than 6,000 patients identified that only $9 \%$ of the trial population were above 70 years of age (12). In addition, fit elderly patients in trials may not represent the general elderly population who are often associated with multiple comorbidities and impaired functional status.

The field of geriatric oncology was developed as there are fundamentally biological and physiological differences between elderly cancer patients and their younger counterparts $(11,12)$. Some of the many physiological changes that distinguish the elderly from the young include: decline in blood flow to the liver, decreased renal clearance and immunosenescence. The decline of blood flow and the reduction of first pass metabolism by the liver can lead to changes in the pharmacokinetics of the cancer drugs (11). Decreased renal clearance due to an age-related decline in renal mass and glomerular filtration rate can affect the clearance of the cancer drugs (11). Immunosenescence which occurs with ageing, is the result of an imbalance between the inflammatory and anti-inflammatory mechanisms leading to a proinflammatory profile causing immune dysregulation (13-15).

In the era of immunotherapy, there are currently no dedicated phase 3 trials on elderly patients to investigate the benefit of immunotherapy. Hence, current evidence is based on subgroup analysis of a few phase 3 trials as shown in Table 1. In a recent journal issue, Nosaki and colleagues (16) report a pooled analysis based on three keynote trials, mainly Keynote 010 (1), Keynote 024 (5) and Keynote 042 (9). Keynote 010 was a phase $2 / 3$ randomized controlled trial of pembrolizumab at 2 and $10 \mathrm{mg} / \mathrm{kg}$ dosages $v s$. docetaxel in previously treated patients with advanced non-small cell lung cancer (NSCLC) with a PD-L1 expression of at least $1 \%$. Primary endpoints were overall survival (OS) and progression free survival (PFS) both in the total population and in patients with PD-L1 expression of at least $50 \%$ of tumour cells. The trial showed that pembrolizumab in both dosages of 2 and $10 \mathrm{mg}$ improved OS as compared 
Table 1 Randomised controlled trials in metastatic NSCLC

\begin{tabular}{|c|c|c|c|}
\hline $\begin{array}{l}\text { Pivotal } \\
\text { NSCLC trials }\end{array}$ & Treatment Arm & Patients $>65$ years & $\begin{array}{l}\text { Subgroup analysis of OS in patients } \\
\qquad 665 \text { years }\end{array}$ \\
\hline \multicolumn{4}{|l|}{ 1st line } \\
\hline Keynote $042(9)$ & Pembrolizumab vs. platinum based chemotherapy & $567(44 \%)$ & NA \\
\hline Keynote 407 (6) & $\begin{array}{l}\text { Pembrolizumab + platinum based chemo vs. platinum } \\
\text { based chemo }\end{array}$ & $305(54 \%)$ & HR $0.63(0.47-0.84)$ \\
\hline Impower 150 (8) & $\begin{array}{l}\text { Atezolizumab+ Bevacizumab (B) and Carboplatin/ } \\
\text { paclitaxel (CP) vs. BCP vs. ACP }\end{array}$ & $359(44.8 \%)$ & NA \\
\hline KeyNote 010 (1) & Pembrolizumab 2 or 10 mg/kg vs Docetaxel & $429(41 \%)$ & HR $0.76(0.57-1.02)$ \\
\hline OAK (4) & Atezolizumab 1,200 mg vs. Docetaxel & $397(47 \%)$ & HR $0.66(0.52-0.83)$ \\
\hline
\end{tabular}

NA, not available.

to docetaxel with a HR, 0.71 (95\% CI: $0.58-0.88)$ and HR, 0.61 (95\% CI: 0.49-0.75) respectively. There was no significant difference in PFS between pembrolizumab and docetaxel. Grade 3-5 treatment related adverse events (TRAEs) were less common with pembrolizumab 2 and $10 \mathrm{mg} / \mathrm{kg}$ as compared to docetaxel at $13 \%$ and $16 \%$ as compared to $35 \%$ respectively. The authors concluded that Keynote 010 confirmed the efficacy and safety of pembrolizumab in previously treated patients with NSCLC and the data supports the $2 \mathrm{mg} / \mathrm{kg}$ dosing given similar outcomes between the two dosages of pembrolizumab.

Keynote 024 (5) was a phase 3 open labeled trial that compared single agent pembrolizumab to chemotherapy in untreated patients with NSCLC with a PD-L1 expression of at least $50 \%$. In Keynote 024 , the primary endpoint was PFS with secondary endpoints including OS and objective response rate. The authors concluded that patients with NSCLC and PD-L1 50\% or greater had significantly longer PFS and OS with pembrolizumab as compared to chemotherapy and that TRAEs grade 3 or greater were less in the pembrolizumab as compared to chemotherapy group at $26.6 \%$ to $53.3 \%$. An updated analysis of Keynote 024 (17) also showed a continual improvement in OS in patients who received pembrolizumab monotherapy as compared to chemotherapy with a median OS of $30.0 \mathrm{vs}$.
14.2 months, HR, 0.63 (95\% CI: 0.47-0.86) respectively. Keynote 042 (9) was a phase 3 trial similar to Keynote 024 except it included patients with PD-L1 of greater than $1 \%$. Primary endpoints were OS in patients with TPS $50 \%$ or greater, TPS $20 \%$ or greater and $1 \%$ or greater. The study showed that overall survival was significantly longer in the pembrolizumab group than in the chemotherapy group in all three TPS populations [ $\geq 50 \%$ : HR, 0.69 (95\% CI: $0.56-$ $0.85), \mathrm{P}=0.0003 ; \geq 20 \%$ : HR, 0.77 (0.64-0.92), $\mathrm{P}=0.002$; $\geq 1 \%$ : HR, 0.81 (0.71-0.93), $\mathrm{P}=0.0018$ ]. TRAEs of grade 3 and more, were less in the pembrolizumab group at $18 \%$ compared to $41 \%$ in the chemotherapy group. An updated analysis of Keynote 042 (18) also showed maintained OS benefit in all subgroups with pembrolizumab as compared to chemotherapy. Importantly, all three Keynote trials included patients that had an Eastern cooperative oncology group performance status (ECOG-PS) of 0-1 and did not have unstable or untreated brain metastasis or carcinomatous meningitis.

The pooled analysis included 2,612 patients of which 264 (10\%) were elderly patients aged 75 years and above (16). Fifteen $(5 \%)$ patients had stable brains metastasis at baseline and 173 (65\%) were treatment naïve. It found that pembrolizumab improved OS for elderly patients with PD-L1 TPS $\geq 1 \%$ with a median OS of 15.7 (10.7- 
20.2) months, HR, 0.76 (95\% CI: 0.56-1.02) when compared to chemotherapy at $11.7(8.4-15.8)$ months. In elderly patients with PD-L1 TPS $\geq 50 \%$, the median OS was 23.1 (11.9-not reached) months, HR, 0.40 (95\% CI: $0.25-0.64)$ as compared to chemotherapy at $8.3(7-11.1)$ months. Similar HRs were noted in the younger population less than 75 years old at HR, 0.76 (95\% CI: 0.69-0.84) in the PD-L1 TPS >1\% and HR, 0.67 (95\% CI: 0.57-0.78) in the PD-L1 TPS $>50 \%$ suggesting that the benefit of pembrolizumab in the elderly is consistent with the overall population. Additionally, safety assessment showed that the incidence of grade 3 and above TRAEs were lower in the patients who received pembrolizumab as compared to chemotherapy at $24.2 \%$ vs. $61 \%$ respectively. Serious TRAEs as defined by life threatening or events that resulted in death or prolong inpatient hospitalisation were noted to be less in the elderly population treated with pembrolizumab as compared to chemotherapy at $16.1 \%$ vs. $26.7 \%$. TRAE leading to discontinuation of treatment was also less in the pembrolizumab group as compared to chemotherapy at $10.7 \%$ vs. $15.2 \%$. TRAEs leading to death were similar in both treatment groups. This pooled analysis is the largest pooled analysis from randomised trials that provide evidence to support the treatment of metastatic lung cancer with ICI in elderly patients 75 years of age and older with an ECOG-PS of 0-1.

Apart from Nosaki et al. (16), Marur and colleagues (19) conducted a similar retrospective pooled analysis of four ICI trials mainly Checkmate 057 (3), Keynote 010 (1), OAK (4) and Poplar (20) in second line setting. The pooled analysis consisted of 2,824 patients of which treatment effect was stratified according to age groups of less than 65,70 years and above, and 75 years and above. In this study, it found 259 patients were 75 years and above and estimated median overall survival was similar across the different age groups at 14.7 (9.1-20.4) months as compared to chemotherapy at $9.5(8.3-15.5)$ months with an age adjusted HR, 0.81 (95\% CI: $0.57-1.14)$. It concluded that patients 75 years and above seem to have the same benefit from ICI although this conclusion may be limited by the small number of patients. Safety assessments showed similar grade 3-4 TRAEs in both subgroups of less than 65 years and 65 years old and greater at $47 \%$ and $49 \%$ respectively but noted a lower incidence of grade 3-4 TRAE at $23 \%$ in patients aged 75 years and older. TRAEs leading to discontinuation of treatment was also similar in the patients aged less than 65 years and 65 years and greater at $7 \%$ while lower in the patients at 75 years and greater at $5 \%$. The authors concluded that the safety assessments by age were exploratory in nature and were limited by the small number of patients $(n=152)$ included in the analysis in patients aged 75 years and greater.

Several conclusions can be made from both the pooled analysis that investigated the efficacy and safety of ICI in elderly patients aged 75 years and older. Firstly, there is an ongoing difficulty in recruiting patients greater than 75 years old despite having more than $40 \%$ of the total trial population to be 65 years or greater. This could be the inherent nature of the elderly population above 75 years that often have multiple co-morbidities and poorer functional status and thus they are not eligible for the stringent trial requirements. Secondly, based on the current pooled analysis, Nosaki and colleagues (17) have provided us with the best evidence to date, to offer treatment to elderly patients who are 75 years and above and fit with an ECOG performance status 0-1. However, extrapolating the results from this study onto the general population of elderly patients needs to be taken with a pinch of salt as real-world elderly patients are often heterogenous and are associated with significant comorbidities and impaired functional status (15).

As such, data from phase 3B/4 trials like Checkmate 153 and real-world data may provide a more realistic assessment of treatment benefit and safety in this group of patients. Checkmate 153 (21) is a phase 3B/4 study of monotherapy nivolumab on previously treated patients with advanced NSCLC. In this study, which has been described to closely reflect the real-world setting, 1,426 patients were recruited of which $556(39 \%)$ were of advanced age of 70 years and above and $128(9 \%)$ had an ECOG PS of 2. Among the patients aged 70 and above, 330 (59\%) had nivolumab as third or later lines similar to the overall population. Median OS across the population was 9.1 months $(95 \%$ CI: 8.3-10.4) while in patients aged 70 and above, median OS was 10.3 months (95\% CI: 8.3-11.5). Both the overall study population and patients aged 70 years and greater had similar 1-year OS at $43 \%$ and $44 \%$ and 2 -year OS rates at $26 \%$ and $25 \%$ respectively. This is in contrast to patients with ECOG PS of 2 who had a poorer median OS of 4.0 months (95\% CI: 3.1-6.2), with 1 and 2-year OS rates of $24 \%$ and $9 \%$ respectively. Reported incidence of grade 3-4 TRAE in the overall population, patients aged 70 years and above and ECOG PS of 2 were at similar rates of $6 \%, 6 \%$ and $9 \%$ respectively. The most common grade 3-4 TRAE in the overall study population and patients aged 70 and greater was fatigue at $2 \%$ and $4 \%$ respectively. In patients with ECOG PS 2, diarrhea and raised alkaline 
phosphatase levels were the highest grade 3-4 TRAE at 2\% individually. Quality of life measures were also included in Checkmate 153. It showed that the patients' quality of life as measured by European Quality of life-5 dimension (EQ-5D) and the lung cancer symptom scale (LCSS) had improved from baseline over the course of treatment with nivolumab. Importantly, in patients who continued to receive treatment, the QOL scores continued to increase close to that of the general population. This improvement was also noted in patients aged $\geq 70$ years while on Nivolumab. In patients with ECOG PS 2, although there was a lower quality of life with a greater symptomatic burden at baseline, improvements in quality of life and decreasing symptoms were noted while undergoing nivolumab treatment.

Khozin et al. (22) recently published a retrospective realworld study based on the US electronic health record data in community cancer clinics in the years following US approval for anti PD-L1 treatment in NSCLC. It showed that out of 1,344 patients, $365(27.2 \%)$ were aged 75 years and older. Six hundred and sixty-nine (49.8\%) patients were treated with PD-1 inhibitors in the second line setting. The estimated median OS was $8.0(6.4-9.5)$ months and neither age at PDL-1 initiation nor stratification by lines of therapy appear to influence OS. Steward and colleagues (23) also published a pilot study to explore the potential use of real-world end points extracted from data from diverse health care data organisations. It included 13,639 patients initiated on PDL1 inhibitors where 4,047 (29.6\%) patients were 75 years and above. In this study, the majority of the patients had anti PD-L1 as a second line treatment. The median OS based on patients aged more than 75 years old ranged from 6.83 to 13.22 months according to the six different datasets.

These findings along with Checkmate 153 showed that efficacy of ICI in the general elderly population seems to be similar to that of the young. Importantly, in elderly patients who had a baseline ECOG PS of 2, where a poor PS is a poor prognostic factor regardless of treatment, the current limited evidence (24) suggests an improvement in quality of life scores with a favourable safety profile. We currently await the results of several ongoing studies like the phase 3 ELDERLY study as well as the phase 4 EPITOP-01 study that would provide further insight into the efficacy and safety in the treatment of ICI in the elderly patients with NSCLC.

\section{Acknowledgments}

None.

\section{Footnote}

Conflicts of Interest: Dr. Kanesvaran received Honorarium from Roche, Astrazeneca, Pfizer, BMS, MSD, Astellas, Janssen, Eisai and Amgen. He is on Advisory board for BMS and MSD. Dr. Chan received Honorarium from Pfizer.

Ethical Statement: The authors are accountable for all aspects of the work in ensuring that questions related to the accuracy or integrity of any part of the work are appropriately investigated and resolved.

\section{References}

1. Herbst RS, Baas P, Kim DW, et al. Pembrolizumab versus docetaxel for previously treated, PD-L1-positive, advanced non-small-cell lung cancer (KEYNOTE-010): a randomised controlled trial. Lancet 2016;387:1540-50.

2. Brahmer J, Reckamp KL, Baas P, et al. Nivolumab versus docetaxel in advanced squamous non-small cell lung cancer. N Engl J Med 2015;373:123-35.

3. Borghaei H, Paz-Ares L, Horn L, et al. Nivolumab versus docetaxel in advanced nonsquamous non-small-cell lung cancer. N Engl J Med 2015;373:1627-39.

4. Rittmeyer A, Barlesi F, Waterkamp D, et al. Atezolizumab versus docetaxel in patients with previously treated non-small-cell lung cancer (OAK): a phase 3, openlabel, multicentre randomised controlled trial. Lancet 2017;389:255-65.

5. Reck M, Rodríguez-Abreu D, Robinson AG, et al. Pembrolizumab versus chemotherapy for PD-L1positive non-small-cell lung cancer. N Engl J Med 2016;375:1823-33.

6. Paz-Ares L, Luft A, Vicente D, et al. Pembrolizumab plus Chemotherapy for Squamous Non-Small-Cell Lung Cancer. N Engl J Med 2018;379:2040-51.

7. Gandhi L, Rodríguez-Abreu D, Gadgeel S, et al. Pembrolizumab plus Chemotherapy in Metastatic Non-Small-Cell Lung Cancer. N Engl J Med 2018;378:2078-92.

8. Socinski MA, Jotte RM, Cappuzzo F, et al. 12 Atezolizumab for First-Line Treatment of Metastatic Nonsquamous NSCLC. N Engl J Med 2018;378:2288-301.

9. Mok TSK, Wu YL, Kudaba I, et al; KEYNOTE-042 Investigators. Pembrolizumab versus chemotherapy for previously untreated, PD-L1-expressing, locally advanced or metastatic non-small-cell lung cancer 
(KEYNOTE-042): a randomised, open-label, controlled, phase 3 trial. Lancet 2019;393:1819-30.

10. National Cancer Institute. Cancer stat facts: lung and Bronchus cancer. Available online: https://seer.cancer.gov/ statfacts/html/lungb.html (accessed October 2019).

11. Marosi C, Köller M. Challenge of cancer in the elderly. ESMO Open 2016;1:e000020.

12. Kanesvaran R, Cordoba R, Maggiore R. Immunotherapy in Older Adults With Advanced Cancers: Implications for Clinical Decision-Making and Future Research. Am Soc Clin Oncol Educ Book 2018;(38):400-14.

13. Quinten C, Coens C, Ghislain I, et al. The effects of age on health-related quality of life in cancer populations: a pooled analysis of randomized controlled trials using the European Organisation for Research and Treatment of Cancer (EORTC) QLQ-C30 involving 6024 cancer patients. Eur J Cancer 2015;51:2808-19.

14. Soto-Perez-de-Celis E, Daneng L, Yuan Y, et al. Functional versus chronological age: geriatric assessments to guide decision making in older patients with cancer. Lancet Oncol 2018;19:e305-16.

15. Ventura MT, Casciaro M, Gangemi S, et al. Immunosenescence in aging: between immune cells depletion and cytokines up-regulation. Clin Mol Allergy 2017;15:21.

16. Nosaki K, Saka H, Hosomi Y, et al. Safety and efficacy of pembrolizumab monotherapy in elderly patients with PDL1-positive advanced non-small-cell lung cancer: Pooled analysis from the KEYNOTE-010, KEYNOTE-024, and KEYNOTE-042 studies. Lung Cancer 2019;135:188-95.

17. Reck M, Rodríguez-Abreu D, Robinson AG, et al. Updated analysis of KEYNOTE-024: pembrolizumab versus platinum-based chemotherapy for advanced nonsmall-cell lung cancer with PD-L1 tumor proportion score of 50\% or greater. J Clin Oncol 2019;37:537-46.

18. Mok TSK Wu YL, Kudaba I, et al. 102OFinal Analysis

Cite this article as: Chan J, Kanesvaran R. The role of immune checkpoint inhibitors (ICI) in the treatment of metastatic non-small cell lung carcinoma in the elderly. Ann Transl Med 2019;7(Suppl 8):S383. doi: 10.21037/atm.2019.12.76 of the Phase III KEYNOTE-042 Study: Pembrolizumab (Pembro) versus Platinum-Based Chemotherapy (Chemo) as First-Line Therapy for Patients (Pts) with PD-L1positive Locally Advanced/Metastatic NSCLC. Ann Oncol 2019;30:ii38-68.

19. Marur S, Singh H, Mishra-Kalyani P, et al. FDA analyses of survival in older adults with metastatic non-small cell lung cancer in controlled trials of PD-1/PD-L1 blocking antibodies. Semin Oncol 2018;45:220-5.

20. Fehrenbacher L, Spira A, Ballinger M, et al. 38 Atezolizumab versus docetaxel for patients with previously treated non-small-cell lung cancer (POPLAR): a multicentre, open-label, phase 2 randomised controlled trial. Lancet 2016;387:1837-46.

21. Spigel DR, McCleod M, Jotte RM, et al. Safety, Efficacy, and Patient-Reported Health-Related Quality of Life and Symptom Burden with Nivolumab in Patients with Advanced Non-Small Cell Lung Cancer, Including Patients Aged 70 Years or Older or with Poor Performance Status (CheckMate 153). J Thorac Oncol 2019;14:1628-39.

22. Khozin S, Abernethy AP, Nussbaum NC, et al. Characteristics of real-world metastatic non-small cell lung cancer patients treated with nivolumab and pembrolizumab during the year following approval. Oncologist 2018;23:328-36.

23. Stewart M, Norden AD, Dreyer N, et al. An exploratory analysis of real-world end points for assessing outcomes among immunotherapy-treated patients with advanced non-small-cell lung cancer. JCO Clin Cancer Inform 2019;3:1-15.

24. Passaro A, Spitaleri G, Gyawali B, et al. Immunotherapy in non-small-cell lung cancer patients with performance status 2: clinical decision making with scant evidence. J Clin Oncol 2019;37:1863-7. 